\title{
Textural development and hydrogen adsorption of carbon materials from PET waste
}

\author{
J.B. Parra ${ }^{\mathrm{a}}$, C.O. Ania ${ }^{\mathrm{a}}$, A. Arenillas ${ }^{\mathrm{a}}$, F. Rubiera ${ }^{\mathrm{a}}$, J.M. Palacios ${ }^{\mathrm{b}}$ and J.J. Pis ${ }^{\mathrm{a} *}$ \\ ${ }^{a}$ Instituto Nacional del Carbón, CSIC, Apartado 73, 33080 Oviedo, Spain \\ ${ }^{b}$ Instituto de Catálisis y Petroleoquímica, CSIC. Campus UAM-Cantoblanco. 28049 Madrid, \\ Spain
}

\begin{abstract}
Polyethyleneterephthalate, PET, has become one of the major post-consumer plastic wastes. PET products present a problem of considerable concern due to the huge amount of solid waste produced. The disposal of this waste, together with its low bio- and photo-degradability represents a serious challenge for industrial countries all over the world. Pyrolysis could provide an alternative and economically viable route for processing PET waste due to the potential uses of different by-products: energy from the pyrolysis gases (58\% yield in this work), recovery of terephthalic acid and other subproducts (20\%), and a solid residue (22\%), which has shown a high textural development after activation. The pyrolysis of PET waste was performed in a quartz reactor (i.d. $35 \mathrm{~mm}$ ) under an inert atmosphere. Further activation was carried out at a temperature of $925^{\circ} \mathrm{C}$, with a flow rate of $10 \mathrm{~mL}$ $\min ^{-1}$ of $\mathrm{CO}_{2}$. A series of carbon materials with different burn-off degrees was obtained. Textural characterisation of the samples was carried out by performing $\mathrm{N}_{2}$ adsorption isotherms at $-196{ }^{\circ} \mathrm{C}$. Changes in the morphological and structural properties of chars were studied by scanning electron microscopy (SEM) and X-ray diffraction (XRD). The carbons obtained were isotropic and highly microporous materials with apparent BET surface areas of up to $2500 \mathrm{~m}^{2} \mathrm{~g}^{-1}$. The suitability of the samples for hydrogen storage was studied by performing $\mathrm{H}_{2}$ adsorption measurements in the 0-1 bar pressure range. Adsorption-desorption experiments showed that reversible physisorption takes place in all the samples. The hydrogen adsorption capacities of the activated PET waste compare favourably well with those attained with high-value carbon materials.
\end{abstract}

Keywords: hydrogen storage, carbon materials, X-ray diffraction 


\section{INTRODUCTION}

Over the years, plastics have become an inextricable part of our society. Having the advantages such as flexibility in design, thermostability, transparency, low cost, and easy to handle, their consumption has grown sharply over the last years. PET consumption in particular has recorded the fastest growth rate in the global plastic market due to the ongoing expansion of the PET bottle market [1]. The use of polyethylene and PET products, as things stand right now, poses a serious environmentally unsustainable problem. This statement stems from the difficulties with post-consumer disposal. Although PET products do not present a direct threat to the environment, they pose a problem of considerable concern due to the huge amount of solid waste produced [2]. The disposal of this waste together with its low bio-degradability and photo-degradability, represents a serious challenge for industrial countries worldwide. The basic challenge is for PET post-consumer reclamation to keep pace with growing consumption.

The most common ways to eliminate PET waste are incineration and recycling [3,4]. Currently, there is a growing interest in PET waste recycling due to the promulgation of more restrictive regulations concerning the recovery of wastes. In addition, the recycling of PET is now a common requirement for both ecological (i.e., considerable amounts of solid waste) and technological reasons (the need to reduce the original raw material consumption). Both incineration and chemical reutilisation techniques produce: i) gaseous products that could pose potential health hazards and, therefore, need to be treated carefully, and ii) residual solid products, which require further disposal [2].

In this work special attention was paid to minimising the potential residues from PET recycling. A number of works have been published on the obtention of active carbons from granulated non-used PET [5], but none concerning the use of PET waste. In addition to the elimination of residual plastic waste, the advantage afforded by the pyrolysis of post-consumer PET is that all the products obtained offer interesting applications (i.e., chemical products, gases with a relatively high heating value and a solid that can be used as a valuable carbon material). The absence of mineral matter and other impurities in the composition of PET waste together with its high carbon content turns this residue into a promising precursor of carbon materials.

The aim of this work was to characterise the texture and structure of the carbon products obtained from PET pyrolysis and subsequent activation in $\mathrm{CO}_{2}$, and to assess their potential as adsorbent materials for novel applications.

One of the possible applications of these materials is hydrogen storage. Hydrogen is currently the object of great interest because it is the cleanest vector of energy. However, the biggest challenge 
facing the transition to hydrogen-based energy systems is the lack of satisfactory hydrogen storage alternatives. Carbon materials have been proposed as a solution for hydrogen storage [6,7]. However, the problems concerning the adsorption capacity and the strength of the interactions between $\mathrm{H}_{2}$ and the carbon materials need to be solved and are currently a subject of wide interest. Most of the studies available in the literature use commercial carbon materials (i.e., commercial activated carbons) or high cost adsorbents (i.e., nanotubes) [8-10].

The main novelty of the present work is based on the utilisation of a residue in order to obtain high-value carbon materials. Thus, the objective is to deal with the problem of plastic disposal and recycling, and, at the same time, assess the efficiency of the adsorbent obtained for its application in hydrogen storage. To attain this goal, the approach followed involved the obtention of the carbon materials, their characterisation and the study of their hydrogen adsorption capacity.

\section{EXPERIMENTAL}

\subsection{Obtention of activated carbons}

The experimental procedure followed during PET waste pyrolysis has been previously described [11]. The char obtained -P- was ground, sieved and a particle size fraction of 0.5-1.0 mm was selected for physical activation with carbon dioxide. First of all, the $\mathrm{P}$ char was treated at $925{ }^{\circ} \mathrm{C}$ under nitrogen atmosphere for one hour (sample PC). Then, batches of around $6 \mathrm{~g}$ of the carbon material were activated in $10 \mathrm{~mL} \mathrm{~min}{ }^{-1}$ of carbon dioxide at $925^{\circ} \mathrm{C}$. Samples with different activation degrees (12, 35, 58 and 76\% burn-off) were obtained and denoted as PC12, PC35, PC58 and PC76, respectively.

\subsection{Characterisation of the activated carbon}

Textural characterisation was carried out by mercury porosimetry and $\mathrm{N}_{2}$ adsorption isotherms at $-196^{\circ} \mathrm{C}$. Different methods (i.e., BET, Dubinin-Radushkevich) were applied to the adsorption isotherms and the resultant textural parameters were compared. The volume and distribution of pores larger than $7.5 \mathrm{~nm}$ were determined with a mercury porosimeter (Carlo Erba Porosimeter 2000). The combination of gas adsorption data and mercury intrusion enables the complete pore volume distribution of the samples to be evaluated.

The pore size distribution can be obtained from the mercury porosimetry data and the t-plot method [12] from $\mathrm{N}_{2}$ adsorption isotherms, using nonporous carbon-coated silica (Sooty Silica) as a reference [13]. The t-plots can provide a break-up of microporosity into narrow microporosity measured from the intercept of the t-plot linear region at lower relative pressure, and into medium microporosity, which is measured from the intercept of the linear region at higher relative pressure. This subdivision, although not in strict accordance with that given by the IUPAC, is widely accepted. 
The samples were crystallographically characterised by means of X-ray diffraction (XRD analysis). The diffractograms were recorded using $\mathrm{Cu} \mathrm{K \alpha}$ radiation $(\lambda=0.15406 \mathrm{~nm})$ at a step size of $0.02^{\circ}$. Additionally, morphological changes of the PET waste during pyrolysis and subsequent activation were evaluated by scanning electron microscopy (SEM).

\subsection{Hydrogen adsorption}

Prior to hydrogen exposure, the samples were outgassed at $300^{\circ} \mathrm{C}$ overnight. Hydrogen adsorption measurements were performed with a Micromeritics ASAP $2010 \mathrm{M}$ at two different temperatures $\left(-196\right.$ and $\left.-186^{\circ} \mathrm{C}\right)$ in the $0-1$ bar pressure range. The hydrogen uptake was calculated from the amount of hydrogen adsorbed on the carbon materials at 1 bar.

Modelling isotherms is a useful tool in adsorption studies, as practical information (i.e., adsorptive capacity) can often be provided. A common approach for describing gas adsorption on surfaces is the Langmuir theory [14]. Although this model derives from a transitional range of adsorption from dilute to a largely covered surface, and does not consider the heterogeneity of the surface of the solid adsorbent, it can satisfactorily describe most Type I isotherms. In this work, therefore, the $\mathrm{H}_{2}$ adsorption data were fitted to the following equation:

$$
\mathcal{V}=\frac{\mathcal{V}_{m} 6 \mathscr{P}}{1+6 \mathscr{P}}
$$

where $\mathcal{V}$ is the volume adsorbed $\left(\mathrm{cm}^{3} \mathrm{~g}^{-1}\right), \mathcal{P}$ is the equilibrium pressure $(\mathrm{mm} \mathrm{Hg}), 6$ is a parameter and $V_{m}$ is the saturated amount adsorbed corresponding to monolayer coverage.

\section{RESULTS AND DISCUSSION}

The samples obtained from PET waste are materials that are mainly composed of carbon [11]. Due to the absence of mineral matter and other impurities in their composition, one possible use might be the fabrication of carbon materials as products of substantial added value.

The effect of the activation process on carbon structure was evaluated by XRD analysis. Typical crystallographic parameters (e.g. height of the crystal(s) or layer(s) of graphitic planes, Lc, and width of these planes, La) can be deduced from the XRD patterns. Figure 1 shows the relationship between these parameters and the burn-off degree of the samples. It can be observed that the activation process produces an increase in the crystallographic parameters of the crystallites, La and Lc. Especially noticeable is the increase in the width of the graphitic planes, La, with burn-off. This could be due to the combination of two different effects that occur during activation. On the one hand the most disordered fraction of the carbon materials is more reactive and, thus, more liable to be destroyed during activation, while only the most ordered structures remain. On the other hand, the higher the 
burn-off degree, the longer the time required at $925{ }^{\circ} \mathrm{C}$ during activation (i.e., 200 min for PC12 and 2600 min for PC76). Thus, some ordering may take place, as a result of which La and Lc increase. However, it should be noted that even with the increase in these parameters, the samples studied in this work did not present highly ordered structures and they are isotropic carbons, as it was evidenced by optical microscopy.

\subsection{Textural development of carbon materials from PET waste}

A detailed textural characterisation of the samples was carried out in order to evaluate their potential fields of applicability. From mercury porosimetry measurements, it was observed that the carbon materials studied are basically non-macroporous. Only PC58 and PC76 present significant intrusion of mercury, as can be observed in Figure 2, indicating that large pores only develop in the final stages of $\mathrm{CO}_{2}$ activation. A SEM micrograph of sample PC76 is presented in Figure 3. The carbon material shows a large number of tunnels and pores of various sizes.

Nitrogen adsorption isotherms at $-196{ }^{\circ} \mathrm{C}$ of the samples were obtained. All the adsorption isotherms were type I according to the BDDT classification [15] indicating that they are mainly microporous materials. The desorption isotherms were found to be reversible, except for sample PC76, which presented a small hysteresis loop. This is indicative of the development of mesoporosity. On the other hand, only the parent material (i.e., PC) exhibits gas retention at low pressures.

The apparent surface area values of the samples were calculated by application of the BET method to the $\mathrm{N}_{2}$ adsorption isotherms. Table 1 shows the apparent BET surface areas along with the textural parameters obtained by application of the Dubinin-Radushkevich -DR- equation. The accessible pore width, L, was calculated from the expression proposed by Stoeckli and Ballerini [16]. It can be observed that as the activation degree increases, there is a clear rise in the amount of nitrogen adsorbed. This indicates that the activation process has induced significant modifications in the texture of the materials. The apparent surface area of the most activated sample (PC76) is nearly four times greater than that of sample PC12 (2468 vs. $668 \mathrm{~m}^{2} \mathrm{~g}^{-1}$ ). In addition, the $\mathrm{C}_{\mathrm{BET}}$ value decreases from 1976 for PC12 to 100 for PC76, indicating that the samples present bigger micropores as the burn-off degree raises. An increase in the micropore volume and the accessible pore width, L, with the burn-off degree was also observed.

It can be seen in Table 1 that the micropore surface area, $\mathrm{S}_{\mathrm{mi}}$, calculated from $\mathrm{N}_{2}$ adsorption isotherm data presents a maximum value. As the pore size becomes larger, the micropore surface area increases up to a determined pore volume, and then decreases. For $\mathrm{N}_{2}$ adsorption, sample PC58 presents the highest micropore surface area.

When the results of the apparent BET surface area and the values obtained from the DR equation are compared, it can be observed that the sample with the lowest burn-off and thus the lowest pore width (i.e., $\mathrm{L}=0.66 \mathrm{~nm}$ ), presents similar surface area values in both methods. However, as the 
burn-off degree increases, and the pore width becomes greater, the surface area increases, especially for the BET method. Sample PC76 presents $870 \mathrm{~m}^{2} \mathrm{~g}^{-1}$ according to DR, while a value of $2468 \mathrm{~m}^{2} \mathrm{~g}^{-1}$ was obtained with the BET method. This result can be attributed to the enlargement of porosity in the most activated samples. The surface areas estimated by the DR method are mainly due to the contribution of the micropores of a smaller size, while the BET method also includes pores of a larger size. Consequently, the enlargement of the micropores with the increase in burn-off degree, leads to a considerable increase in BET surface area compared to the values obtained from the DR method.

Figure 4 shows the evolution of the micropore size distribution with the activation degree of the samples, according to the Horvath-Kawazoe method [17], which was applied to the nitrogen adsorption isotherms. It can be observed that sample PC12 presents only one peak at around $0.7 \mathrm{~nm}$, in accordance with the results from the Dubinin-Radushkevich equation (see Table 1). The results also show that the higher the activation degree of the sample, the wider the micropore size distribution. The height of the maximum at $0.7 \mathrm{~nm}$ decreases as the larger micropores appear.

Tailoring the pore size of a carbon material is of considerable importance for adsorption applications. It should be noted that, in the materials obtained, the surface areas values are highly significant and comparable to commercial active carbons. In addition, it is possible by controlling the micropore size distribution to obtain carbon materials with adequate textural properties in accordance with their specific applications. Moreover, the source of these carbon materials (i.e., PET waste) gives added relevance to the results.

\subsection{Hydrogen adsorption}

As a consequence of the growing interest in hydrogen technology in recent years, one possible application of the materials obtained from PET waste could be hydrogen storage. The suitability of the obtained carbon materials for hydrogen storage was studied by performing $\mathrm{H}_{2}$ adsorption measurements in the 0-1 bar pressure range. The adsorption isotherms at -196 and $-186{ }^{\circ} \mathrm{C}$ are displayed in Figure 5. Adsorption-desorption experiments showed that reversible physisorption takes place in all the samples.

It can be seen that at low pressures the adsorption of $\mathrm{H}_{2}$ decreases with the increase in burn-off at both temperatures. However, at higher pressures, the adsorption isotherm for PC12, which showed the highest $\mathrm{H}_{2}$ adsorption at lower pressures, intersects other adsorption isotherms and shows lesser adsorption. The $\mathrm{H}_{2}$ adsorption on the rest of the samples moves steadily upwards, and the uptake increases with the burn-off degree. The results of these $\mathrm{H}_{2}$ physisorption measurements are given in Table 2. The values obtained for the samples studied in this work are comparable to or, in many cases, better than other results obtained with higher cost carbon materials under the same experimental conditions $[18,19]$. 
Hydrogen adsorption isotherms follow practically the pattern of Langmuir adsorption. The fitting of the experimental data to the Langmuir equation results in the evaluation of the maximum adsorption capacity (cf. Table 2). The measured and the calculated data are similar for PC12, although the calculated values are somewhat higher for the samples with higher degrees of activation. In the light of these results, it can be assumed that a hydrogen monolayer covers the sample PC12 at 1 bar, whereas on the most activated samples, the hydrogen adsorbed molecules do not complete the monolayer.

In a first approach the total amount of hydrogen taken up per gram of adsorbent at both temperatures was correlated with the apparent BET surface area, and the pore volumes of the activated samples (Figure 6). The increase in surface area and micropore volume -calculated from the DR equation applied to the nitrogen adsorption data- gave rise to an increase in $\mathrm{H}_{2}$ adsorption capacity. The good correlation between the surface area of the samples and the uptake of hydrogen suggests that the $\mathrm{H}_{2}$ adsorption on the carbon materials depends primarily on their textural properties. Thus, it seems that hydrogen is basically physisorbed (non-dissociatively adsorbed) on the carbon structure of the samples studied. This is also corroborated by the reversibility of the adsorption isotherms.

Some authors have pointed out that high microporosity does not always give rise to a corresponding increase in hydrogen capacity and that hydrogen is not exclusively adsorbed in the micropores but also on the surface of the mesopores [19]. In addition, recent studies have concluded that carbon materials must have a narrow and adequate pore size distribution for a strong interaction with hydrogen at room temperatures and at moderate pressures [8]. The results obtained in the present work are in accordance with the above findings. In this respect, it can be seen in Figure 6 that a good correlation between hydrogen adsorption capacity and the volumes of medium-micropores and mesopores (calculated from the t-plot method) is attained. This correlation, however, is not straightforward regarding the narrow microporosity. These results point out that large storage capacities are obtained in the samples containing large volumes of micropores of adequate size. A contribution of the uptake, therefore, might also be due to the generation of mesoporosity in the most activated samples (PC58 and PC76).

According to the ideal Langmuir model, there is a relation between the average potential energy of the adsorption process and the heat of adsorption. Knowledge concerning the magnitude of isosteric heat of adsorption and its variation with coverage can provide useful information regarding the nature of the surface and the adsorbed phase, along with the contribution of the forces involved in physical adsorption (i.e., van der Waals forces and electrostatics interaction comprising polarisation, dipole and quadripole interactions). Then, the calculation of adsorption energies allows the estimation of the nature of the forces in the contribution to the adsorption potential. The isosteric heat of adsorption $\left(\Delta \mathrm{H}_{\mathrm{isot}}\right)$ was determined as a function of the degree of surface coverage $\vartheta$ using the Clausius-Clapeyron equation: 


$$
\frac{\Delta H_{\text {isost }}}{R}=\left(\frac{\partial \ln P}{\partial(1 / T)}\right)_{\vartheta}
$$

where $\mathrm{R}$ is the universal gas constant in $\mathrm{kJ} \mathrm{K}^{-1} \mathrm{~mol}^{-1}, \mathrm{P}$ the pressure in torr, $\mathrm{T}$ the analysis temperature in degrees $\mathrm{K}$ and $\vartheta$ the degree of surface coverage calculated using the value of monolayer capacity determined from the BET analysis of the sample. The isotherms pressure range used in the calculation of the isosteric heat of adsorption was chosen to be 0.03-1bar and the coverage ranged from 0.005 up to 1 . Figure 7 shows the variation of the isosteric heat of adsorption of the activated samples with coverage.

From Figure 7 it is inferred that the heat of adsorption is dependent on coverage, which states that the effects of surface heterogeneity are significant in the samples studied. A large increase in $\Delta \mathrm{H}_{\text {isot }}$ at low coverage was observed. On the other hand, as the coverage increases and the saturation limit is approached, $\Delta \mathrm{H}_{\text {isot }}$ decreases and remains almost constant. This effect is especially noticeable in sample PC12 implying that the hydrogen monolayer was only completed for PC12 sample.

This underlines the effect of the electrostatic contribution (comprising polarisation and dipole and quadrupole interactions) to the adsorption energy. At low coverage, hydrogen molecules are adsorbed at the available active sites in the carbonaceous material to form stable species; the high heat of adsorption indicates the strong bonding of hydrogen to the carbon surface. The contribution due to interactions between neighbouring adsorbed molecules is negligible and the increase in the heat of adsorption can only be attributed to hydrogen-adsorbent electrostatic interactions.

At the same time, at a higher coverage, the heat of adsorption remains almost constant, suggesting that the electrostatic contribution is minor and that the effect of intermolecular attraction forces between neighbouring adsorbed molecules is not significant. The surface appears to be almost energetically uniform to hydrogen adsorption at large coverage, when all the active sites are already covered.

The results of the isosteric heat of adsorption, suggest that a larger number of adsorption sites with similar adsorption energies are present in the most activated samples. The most uniform adsorption energies were found for the samples with the highest degree of burn-of. This is in good agreement with the structure of the materials, concerning the textural characteristics mentioned above. For instance, sample PC12 showed the largest energetic surface heterogeneity, which is in good agreement with its low apparent surface area and its poorly developed microporosity.

It was observed that the increase in the activation degree produces a gradual increase in the volume of total micropores, due to an enlargement of the small micropores as evidenced by the $\mathrm{L}$ parameter (see Table 1). This enlargement of narrow microporosity with the degree of burn-off might be responsible for the gradual energetic homogeneity of the activated samples. The extent to which an 
adsorbent appears energetically heterogeneous depends, to some extent, on the size of the sorbate molecule, and the pore size distribution of the adsorbent [20]. The surface of PC12, therefore, might appear energetically heterogeneous, since the active sites might not be accessible to hydrogen, due to the narrow porous structure of sample PC12. An increase in burn-off degree gives rise to an enlargement of the porous structure of the samples, and thus, all favourable sites are extended over regions accessible to the adsorbate. When active sites are accessible, hydrogen adsorbed molecules have the potential of adsorption averaged over a larger region and thus the surface appears almost energetically uniform.

\section{CONCLUSIONS}

Carbon materials with high apparent BET surface areas (i.e., $2500 \mathrm{~m}^{2} \mathrm{~g}^{-1}$ ) and mainly microporous have been obtained from the physical activation of PET waste. Highly activated samples also presented noteworthy mesoporosity.

The carbon materials obtained from PET waste pyrolysis and $\mathrm{CO}_{2}$ activation seem to have suitable textural properties (high surface area and large micropore volume) for hydrogen uptake. The adsorption process at the temperatures studied is considered to be non-dissociative since no hysteresis is present in the uptake-release process. The maximum hydrogen uptake was calculated to be $2.3 \%$ for the most activated sample (PC76) at $-196{ }^{\circ} \mathrm{C}$. These results are quantitatively higher than those attained with high-cost carbon adsorbents (i.e., nanotubes) accomplished by other authors under the same conditions.

The adsorption isotherms were well described by the Langmuir model. However, the results from the isosteric heat of adsorption revealed the presence of heterogeneous adsorption sites on the samples at low coverage. The degree of energetic heterogeneity at low coverage was found to be larger for the less activated samples. This is in good agreement with the textural characteristics developed in the samples as a consequence of the activation process of the PET waste.

An increase in the activation degree of PET waste produces a gradual enhancement in the volume of total micropores due to an enlargement of the small micropores. This enlargement of small microporosity might be responsible for the easier accessibility of hydrogen molecules to active sites in highly activated samples. Thus, when active sites are accessible to hydrogen molecules, the potential of adsorption is averaged over a larger region and the surface appears energetically homogeneous.

\section{REFERENCES}

1. Association of plastic manufacturers in Europe, "An analysis of plastics consumption and recovery in Western Europe”, Spring 2001

2. P.S. Bhandare, B.K. Lee, K. Krishnan, J. Thermal Anal. 49 (1997) 361-370.

3. K.S. Rebeiza, A.P. Craftb, Resour. Conserv. Recycl. 15 (1995) 245-257. 
4. S. Mishra, A.Z. Goje, V.S. Zope, Polymer Reaction Eng. 11 (2003) 79-99.

5. K. Laszló, A. Szucs, Carbon 39 (2000) 1945-1953.

6. F.L. Darkim, P. Malbrunot, G.P. Tartaglia, Int. J. Hydrogen Energy 27 (2002) 193-202.

7. X. Chen, U.D. Weglikowska, M. Haluska, M. Hulman, M. Hirscher, M. Becher, S. Roth, Mat. Res. Soc. Symp. Proc. 706 (2002) 11.

8. A.C. Dillon, M.J. Heben, Applied Physics A Mat. Sci. Proc. 72 (2001) 133-142.

9. E. Johansson, B. Hjörvarsson, T. Ekström, M. Jacob, J. Alloys Comp. 330-332 (2002) 670675.

10. R. Ströbel, L. Jörissen, T. Schliermann, V. Trapp, W. Schütz, K. Bohmhammel, G. Wolf, J. Garche, J. Power Sources 84 (1999) 221-224.

11. J.B. Parra, C.O. Ania, A. Arenillas, J.J. Pis, Stud. Surf. Sci. Cat. 144 (2002) 537-544.

12. B.C. Lippens, J.H. de Boer, J. Catalysis 4 (1965) 319-323.

13. P.J.M. Carrot, R.A. Roberts, K.S.W. Sing, Carbon 25 (1987) 769-770.

14. I. Langmuir, J. Amer. Chem. Soc. 40 (1918) 1361-1403.

15. S. Brunauer, L.S. Deming, W.E. Deming, E. Teller, J. Am. Chem. Soc. 62 (1940) 1723-1732.

16. F. Stoeckli, L. Ballerini, Fuel 70 (1991) 557-560.

17. G. Horvath, K. Kawazoe, J. Chem. Eng. Japan 16 (1983) 470-475.

18. R. Chahine, P. Bénard, IEA. Ed. Sandrock G. SunaTech Inc. (2001) 104-107.

19. M.G. Nijkamp, J.E.M.J. Raaymakers, A.J. van Dillen, K.P. de Jong, Applied Physics A Mat. Sci. Proc 72 (2001) 619-623.

20. D.M. Ruthven, "Principles of adsorption and adsorption process”. Wiley \& Sons, New York, 1984. 


\section{LIST OF TABLES}

Table 1. Textural properties, from $\mathrm{N}_{2}$ adsorption isotherms at $-196{ }^{\circ} \mathrm{C}$, of the samples studied

Table 2. Hydrogen adsorption capacities at -196 and $-186^{\circ} \mathrm{C}$ and 1 bar for different carbonaceous materials, and Langmuir fitting parameters. 
Table 1. Textural properties, from $\mathrm{N}_{2}$ adsorption isotherms at $-196{ }^{\circ} \mathrm{C}$, of the samples studied

\begin{tabular}{|c|c|c|c|c|c|c|c|c|c|c|}
\hline & \multirow[b]{2}{*}{$\begin{array}{c}\mathrm{S}_{\mathrm{BET}} \\
\left(\mathrm{m}^{2} \mathrm{~g}^{-1}\right)\end{array}$} & \multirow[b]{2}{*}{$\mathrm{C}_{\mathrm{BET}}$} & \multicolumn{4}{|c|}{ DR equation } & \multicolumn{3}{|c|}{ t-plot method } & \multirow[b]{2}{*}{$\begin{array}{c}V_{\mathrm{ma}}^{\mathrm{d}} \\
\left(\mathrm{cm}^{3} \mathrm{~g}^{-1}\right)\end{array}$} \\
\hline & & & $\begin{array}{c}\mathrm{W}_{0} \\
\left(\mathrm{~cm}^{3} \mathrm{~g}^{-1}\right)\end{array}$ & $\begin{array}{c}\mathrm{E}_{\mathrm{o}} \\
\left(\mathrm{kJ} \mathrm{mol}^{-1}\right)\end{array}$ & $\begin{array}{c}\mathrm{L} \\
(\mathrm{nm})\end{array}$ & $\begin{array}{c}\mathrm{S}_{\mathrm{mi}} \\
\left(\mathrm{m}^{2} \mathrm{~g}^{-1}\right)\end{array}$ & $\begin{array}{c}\mathrm{V}_{\mathrm{nmi}}{ }^{\mathrm{a}} \\
\left(\mathrm{cm}^{3} \mathrm{~g}^{-1}\right)\end{array}$ & $\begin{array}{c}\mathrm{V}_{\mathrm{mmi}}{ }^{\mathrm{b}} \\
\left(\mathrm{cm}^{3} \mathrm{~g}^{-1}\right)\end{array}$ & $\begin{array}{c}V_{\mathrm{me}}{ }^{\mathrm{c}} \\
\left(\mathrm{cm}^{3} \mathrm{~g}^{-1}\right)\end{array}$ & \\
\hline PC12 & 668 & 1976 & 0.25 & 27.8 & 0.66 & 629 & 0.183 & 0.075 & 0.008 & 0.013 \\
\hline PC35 & 1405 & 532 & 0.52 & 21.8 & 1.04 & 909 & 0.263 & 0.290 & 0.041 & 0.017 \\
\hline PC58 & 1920 & 203 & 0.67 & 19.0 & 1.42 & 930 & 0.266 & 0.527 & 0.121 & 0.029 \\
\hline PC76 & 2468 & 100 & 0.90 & 16.3 & 2.19 & 870 & 0.268 & 0.819 & 0.236 & 0.062 \\
\hline
\end{tabular}

${ }^{\mathrm{a}} \mathrm{V}_{\mathrm{nmi}}$, volume of narrow micropores (pore diameter $\mathrm{w}<0.7 \mathrm{~nm}$ )

${ }^{\mathrm{b}} \mathrm{V}_{\mathrm{mmi}}$, volume of medium micropores (pore diameter $0.7<\mathrm{w}<2 \mathrm{~nm}$ )

${ }^{\mathrm{c}} \mathrm{V}_{\mathrm{me}}$, volume of mesopores (pore diameter $2<\mathrm{w}<50 \mathrm{~m}$ )

${ }^{\mathrm{d}} \mathrm{V}_{\mathrm{ma}}$, volume of macropores from mercury porosimetry (pore diameter $\mathrm{w}>50 \mathrm{~nm}$ ) 
Table 2. Hydrogen adsorption capacities at -196 and $-186^{\circ} \mathrm{C}$ and 1 bar for different carbon materials, and Langmuir fitting parameters.

\begin{tabular}{|c|c|c|c|c|c|c|c|c|c|}
\hline Sample $^{\mathrm{a}}$ & $\begin{array}{c}\mathrm{S}_{\mathrm{BET}} \\
\left(\mathrm{m}^{2} \mathrm{~g}^{-1}\right)\end{array}$ & $\begin{array}{c}\mathrm{H}_{2} \\
\text { (wt\%) }\end{array}$ & $\begin{array}{c}\mathcal{V} m \\
\text { (wt \%) }\end{array}$ & 6 & $r^{2}$ & $\begin{array}{c}\mathrm{H}_{2} \\
(\mathrm{wt} \%)\end{array}$ & $\begin{array}{c}V m \\
\text { (wt \%) }\end{array}$ & 6 & $r^{2}$ \\
\hline & & \multicolumn{4}{|c|}{ Adsorption at $-196{ }^{\circ} \mathrm{C}$} & \multicolumn{4}{|c|}{ Adsorption at $-186^{\circ} \mathrm{C}$} \\
\hline PC12 & 668 & 1.4 & 1.6 & 0.010 & 0.9993 & 1.2 & 1.5 & 0.005 & 0.9995 \\
\hline PC35 & 1405 & 1.9 & 2.2 & 0.007 & 0.9985 & 1.5 & 1.8 & 0.005 & 0.9971 \\
\hline PC58 & 1920 & 2.1 & 2.8 & 0.004 & 0.9993 & 1.6 & 2.3 & 0.003 & 0.9989 \\
\hline PC76 & 2468 & 2.3 & 3.2 & 0.003 & 0.9986 & 1.7 & 2.6 & 0.002 & 0.9983 \\
\hline AC & 1100 & 1.9 & \multicolumn{3}{|c|}{ Reference [18] } & & & & \\
\hline SWNT & 266 & 0.6 & \multicolumn{3}{|c|}{ Reference [18] } & & & & \\
\hline $\mathrm{CNF}$ & 238 & 0.2 & \multicolumn{3}{|c|}{ Reference [19] } & & & & \\
\hline
\end{tabular}

${ }^{\mathrm{a} A C}$ : activated carbon; SWNT: single walled nanotube; CNF: carbon nanofibres. 


\section{List of Figures}

Figure 1. Relationship between the burn-off degree of the samples and the crystallographic parameters, La and Lc, obtained from XRD.

Figure 2. Cumulative pore volume curves obtained from mercury porosimetry.

Figure 3. SEM micrograph of the PC76 sample.

Figure 4. Micropore size distribution of the samples studied using the Horvath-Kawazoe method.

Figure 5. Hydrogen adsorption isotherms at a) -196 and b) $-186^{\circ} \mathrm{C}$.

Figure 6. Correlation of hydrogen adsorption capacities with the apparent surface area and porosity of the samples studied. a. -196 and b. $-186^{\circ} \mathrm{C}$.

Figure 7. Isosteric heat of hydrogen adsorption for the samples obtained from PET waste 
Figure 1. Relationship between the burn-off degree of the samples and the crystallographic parameters, La and Lc, obtained from XRD.

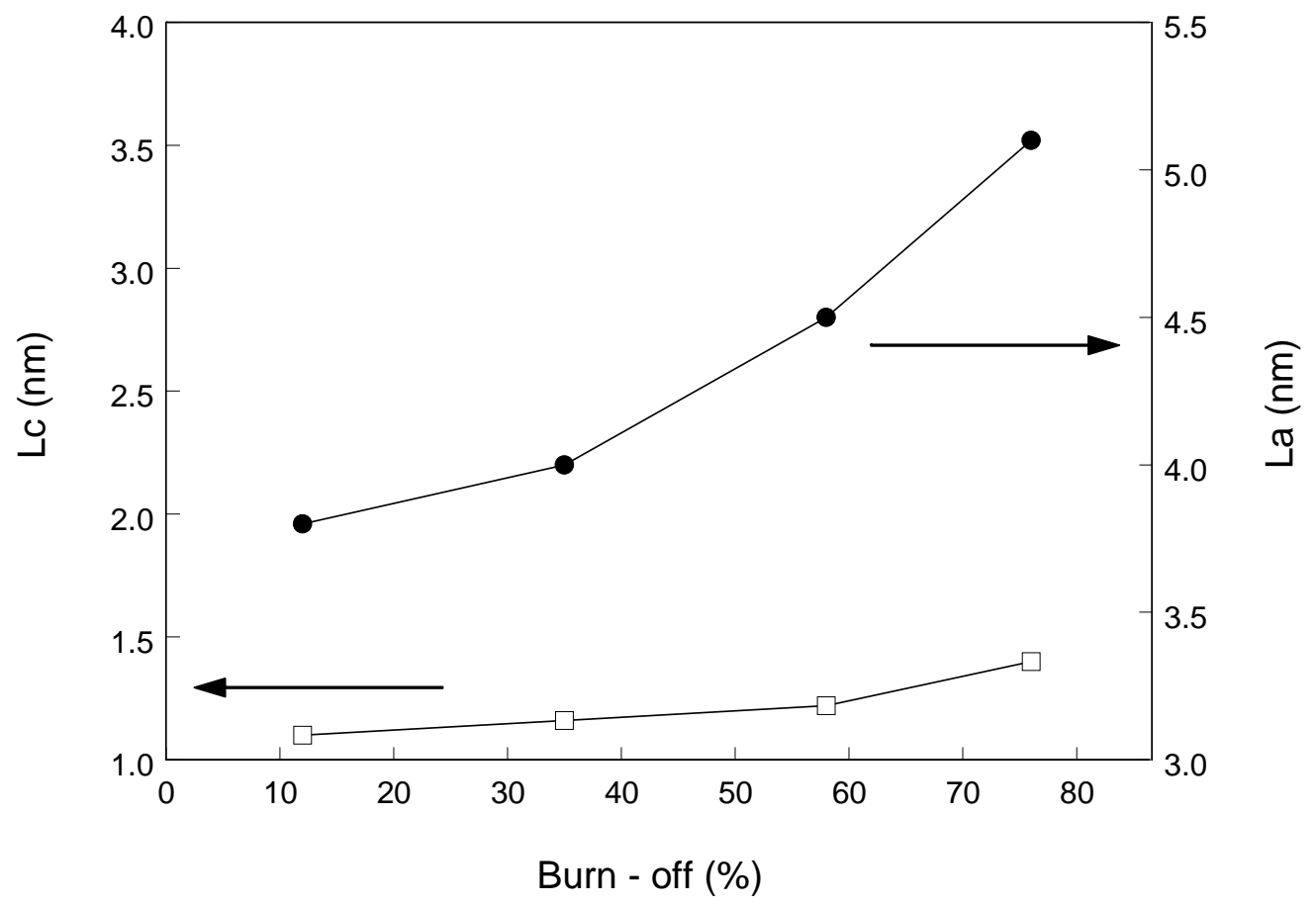


Figure 2. Cumulative pore volume curves obtained from mercury porosimetry.

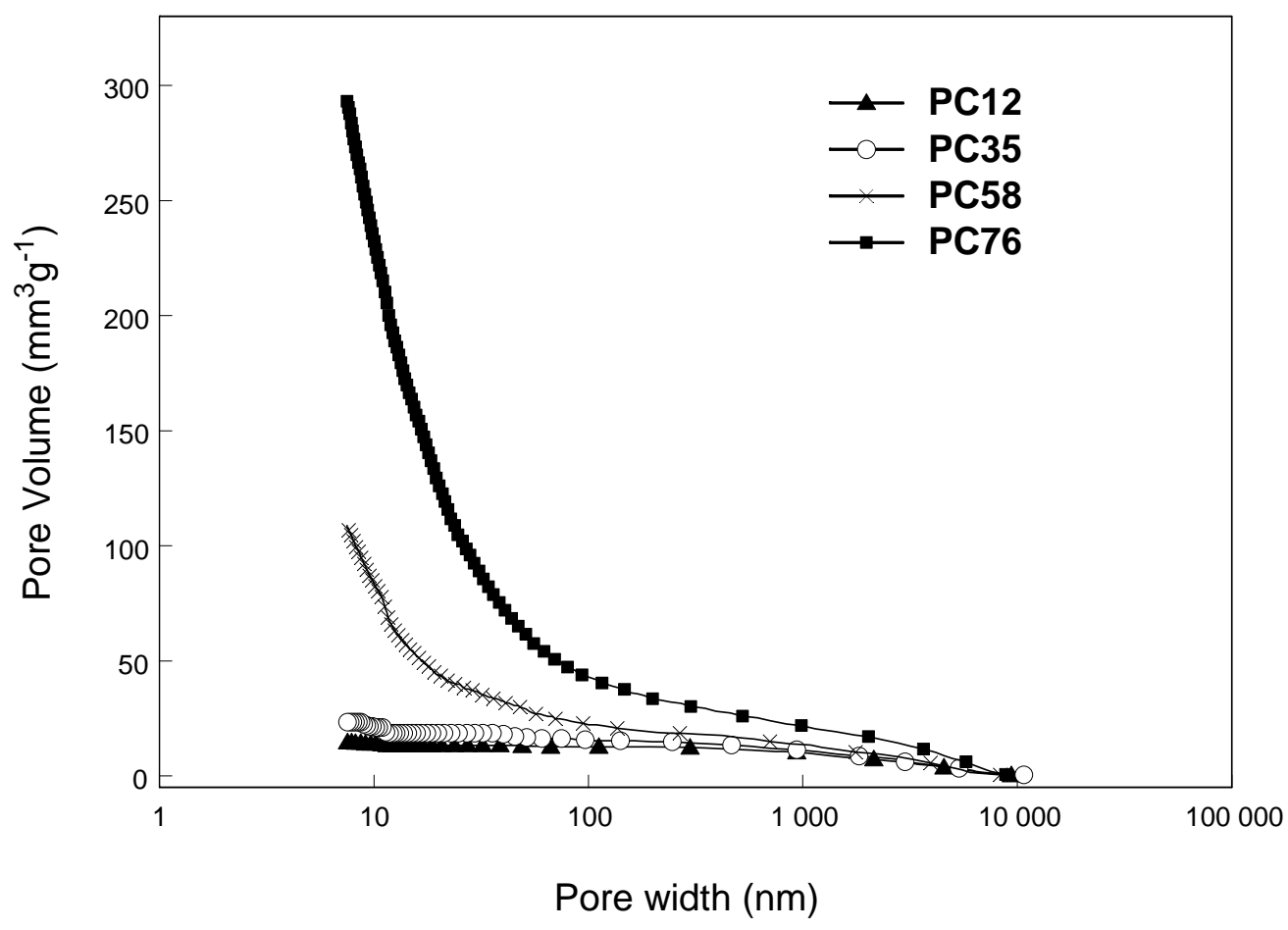


Figure 3. SEM micrograph of the PC76 sample.

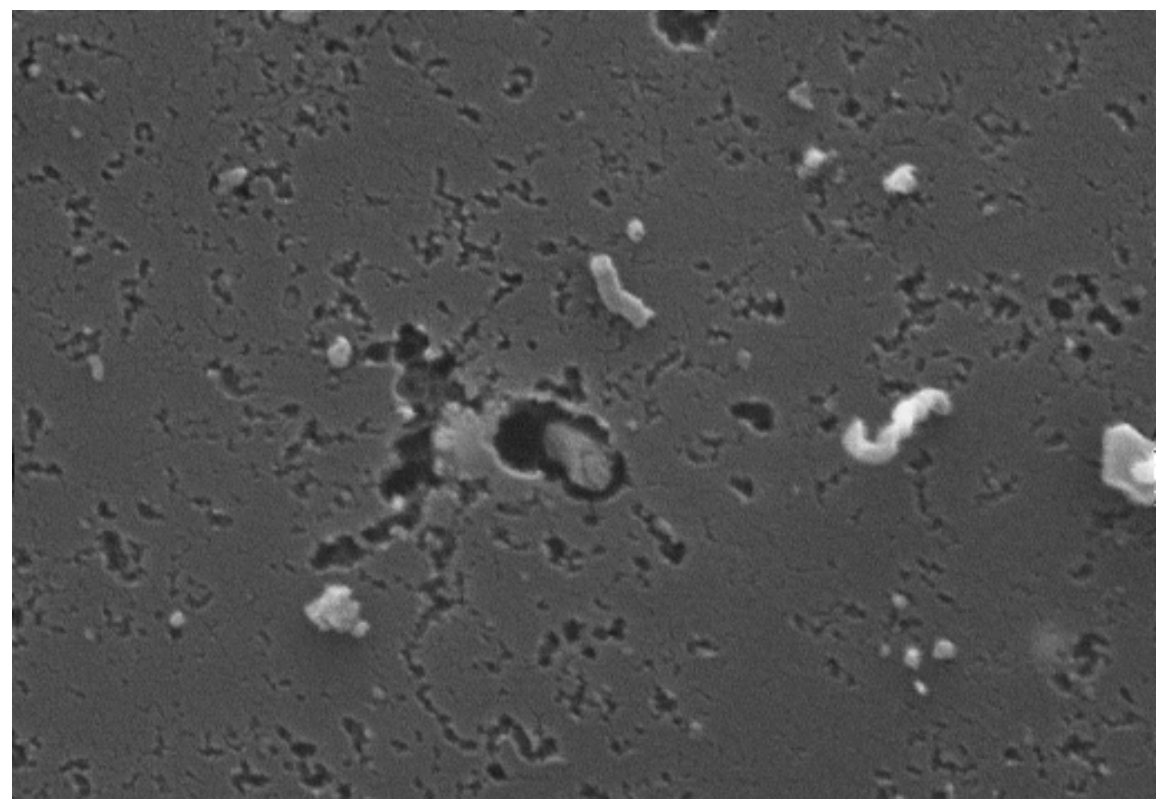


Figure 4. Micropore size distribution of the samples studied using the Horvath-Kawazoe method.
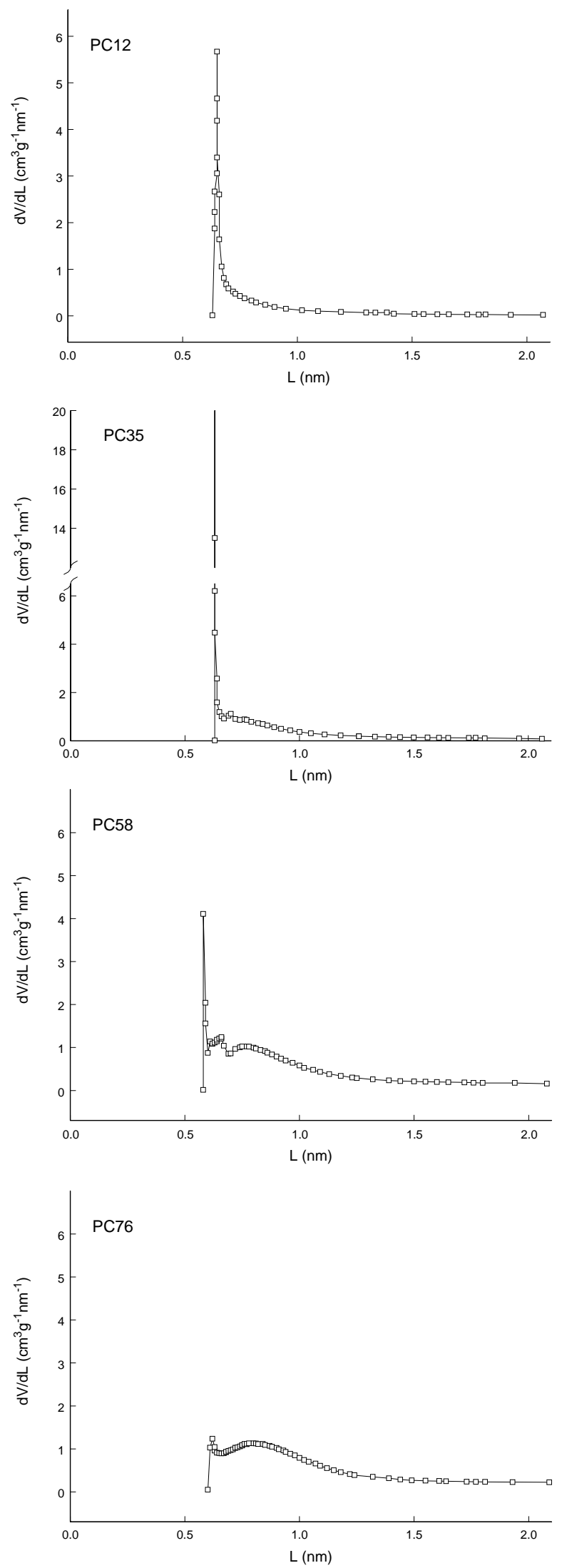
Figure 5. Hydrogen adsorption isotherms at a) -196 and b) $-186^{\circ} \mathrm{C}$.
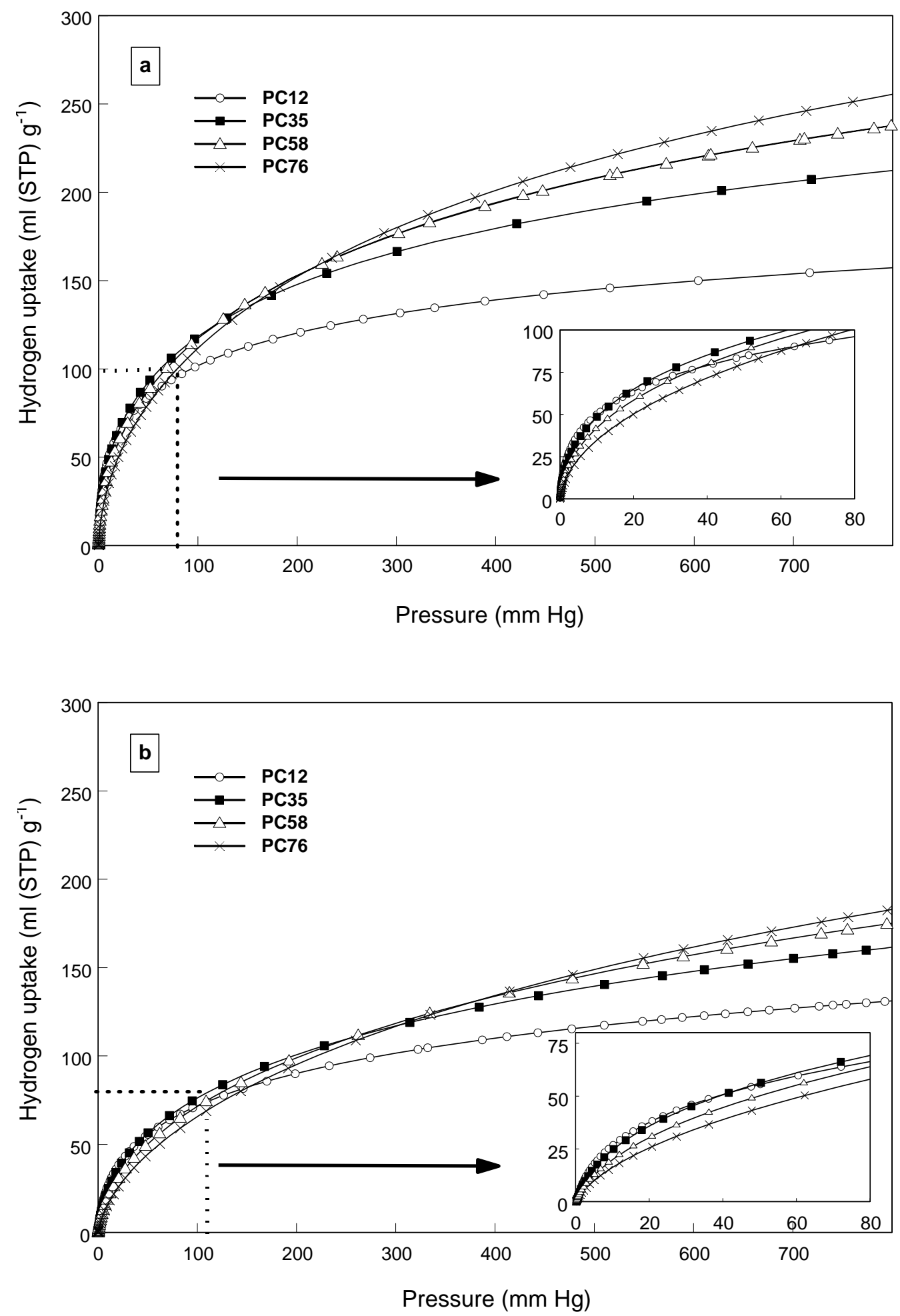
Figure 6. Correlation of hydrogen adsorption capacities with the apparent surface area and porosity of the samples studied. a) -196 and b) $-186^{\circ} \mathrm{C}$.
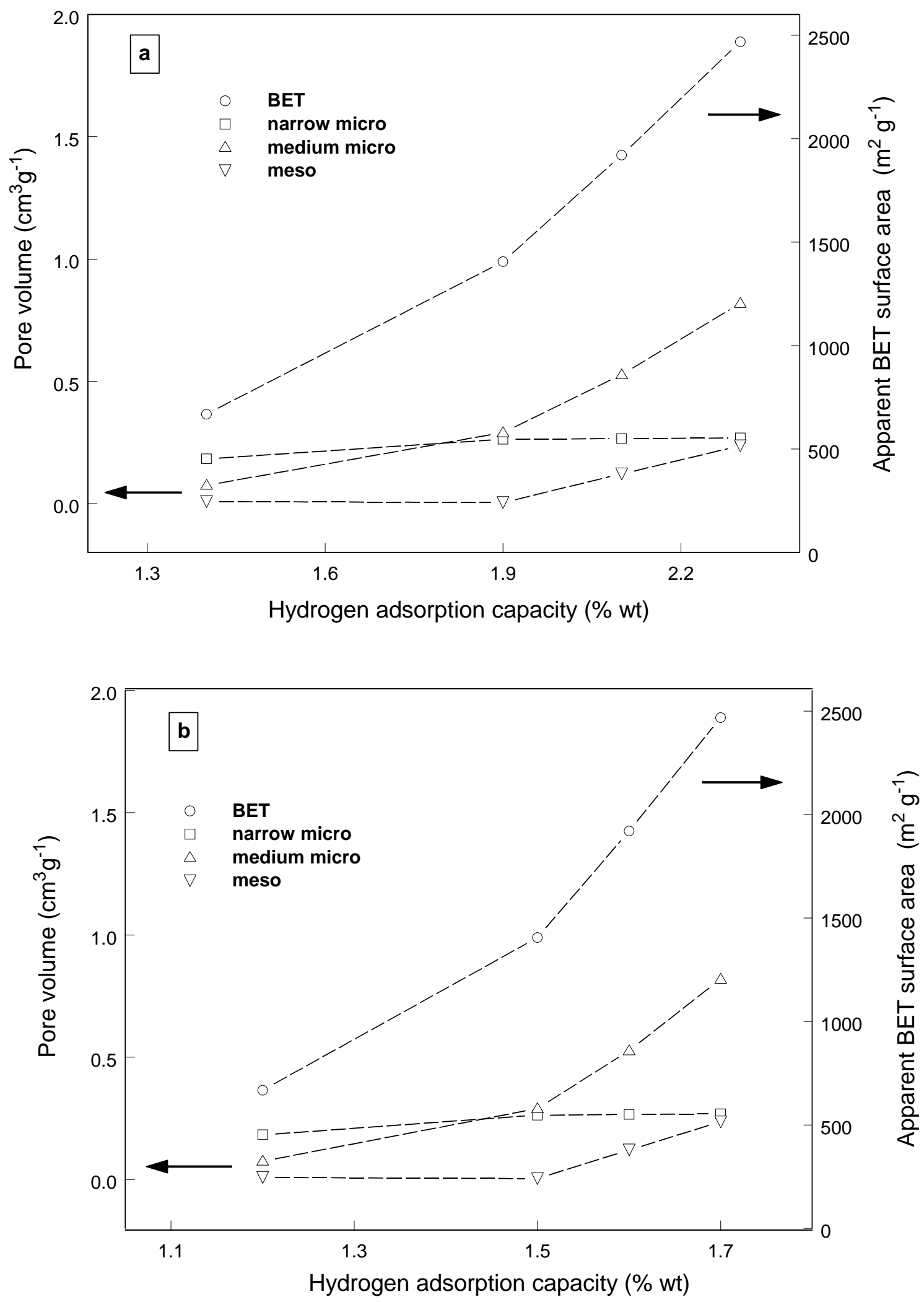
Figure 7. Isosteric heat of hydrogen adsorption for the samples obtained from PET waste

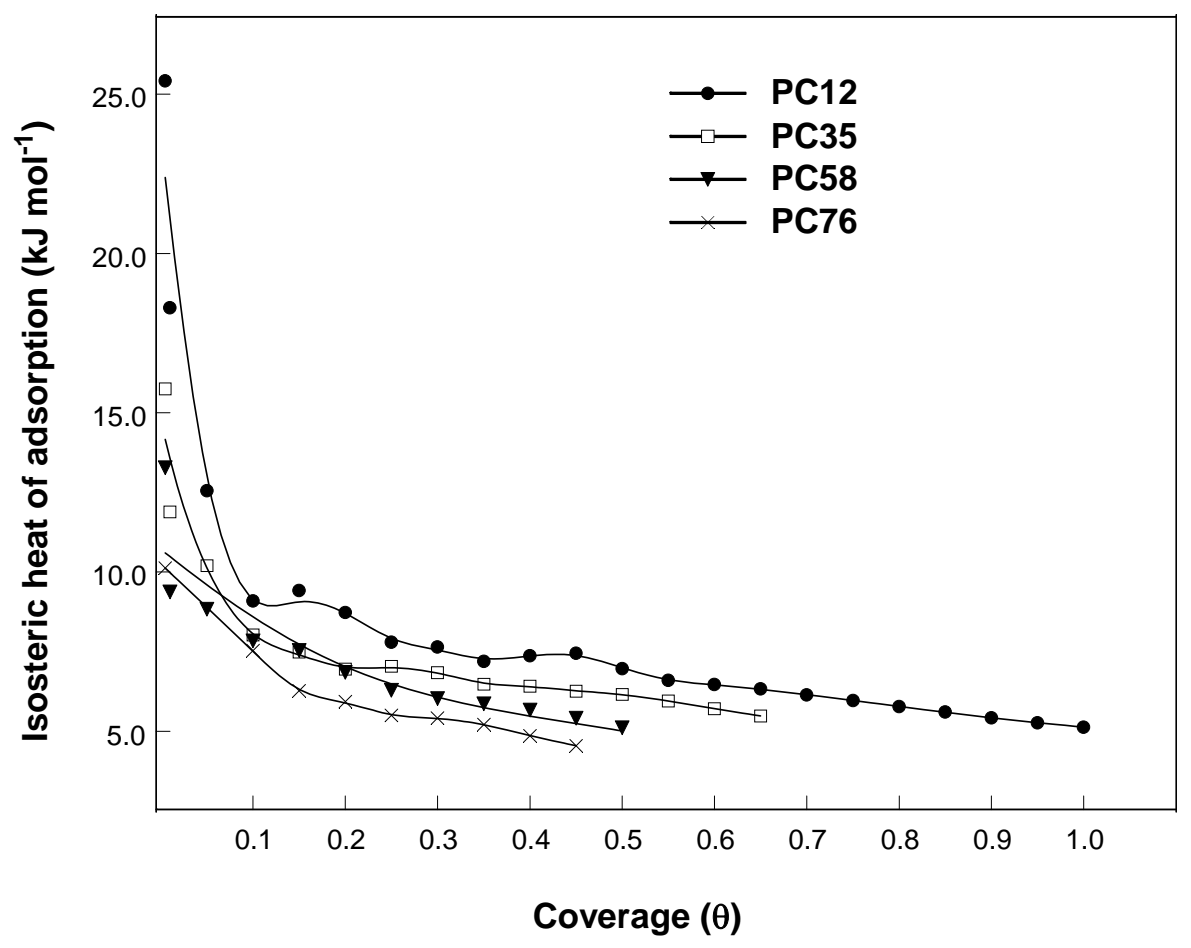

